\title{
Dynamic frameworks: the role of non-covalent interactions
}

\author{
S.A. Bourne \\ Centre for Supramolecular Chemistry Research, Department of Chemistry, University of Cape Town, Rondebosch 7701, South Africa
}

Susan.Bourne@uct.ac.za

Supramolecular chemistry places focus on the weak non-covalent interactions between molecules in the solution or solid phases. These "soft" interactions are reversible and allow one to build materials which are responsive to their chemical or physical environment, changing form and properties under the influence of heat, light, pressure, or chemical probes.

Dynamic materials, capable of responding to their environment, require flexibility which may be achieved using interactions such as hydrogen- or halogen-bonding or through the use of suitable metals and ligands in coordination compounds. Frameworks may be made up of relatively strongly bound entities such as those that make up metal-organic frameworks (MOFs) or may be more loosely bound such as host-guest systems where the host molecules crystallise as independent entities but leave spaces which can accommodate guest molecules. The process of guest exchange within porous solids can be used in a range of applications, such as selective absorption or separation of gases and heterogeneous catalysis. Among the more interesting examples of dynamic processes in frameworks are those which result in thermochromic and/or mechanochromic effects. Materials of this type are particularly of interest if they are able to revert to their original state on application of another external perturbation signal. Rational design of such systems remains a challenge however, and is thus an exciting area for application of crystal engineering principles.

In this presentation, examples from recent work in our laboratory will be presented, including MOFs and 3D hydrogen bonded frameworks constructed from the same flexible ditopic ligands. The influence of halogen versus hydrogen bonding on a molecular host-guest system will also be described. Frameworks exhibit thermochromic and mechanochromic properties, depending on the application of external stimuli such as heat, grinding or exposure to solvent vapours. Further examples will include the selective inclusion of halogenated volatile organic compounds in a porous metal-organic framework (Fig. 1).

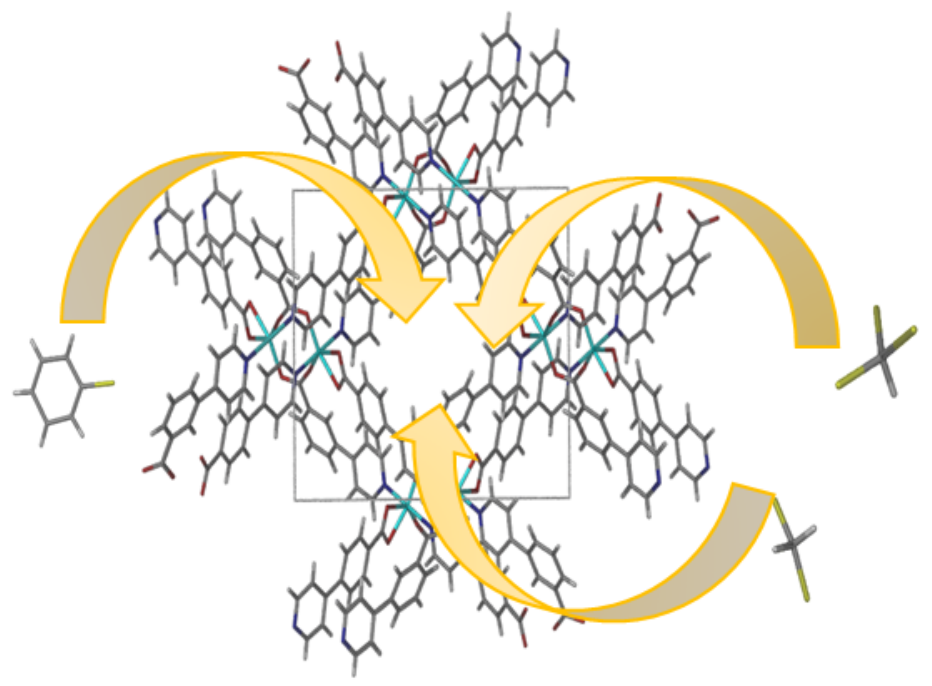

Figure 1. Selective inclusion of halogenated volatile organic compounds in a porous metal-organic framework made using mixed ditopic ligands.

Keywords: frameworks; hydrogen bonding; halogen bonding; guest exchange; chromism 